\title{
Spinal cord stimulation with percutaneous and plate electrodes: side effects and quantitative comparisons
}

\section{Richard B. North, M.D., Amy Lanning, Ryan Hessels, and Protagoras N. Cutchis, M.D.}

Department of Neurosurgery, School of Medicine, and Applied Physics Laboratory, Johns Hopkins University, Baltimore, Maryland

Spinal cord stimulation is limited by the uncomfortable side effects experienced by the patient as the amplitude of stimulation is increased. These side effects include local segmental paresthesias or motor responses, which are objectively demonstrable as frequency-following muscle contractions, attributable to dorsal root stimulation. The authors present evidence for another mechanism of stimulation-evoked discomfort, namely recruitment of small fibers in ligamentum flavum, which occurs when electrodes are inserted percutaneously and their contacts are exposed circumferentially; this does not occur with plate electrodes with insulated dorsal surfaces.

In a consecutive series of 79 patients with postsurgical lumbar pain syndromes, percutaneous four-contact electrodes were tested at levels from T-8 through T-12. At one or more levels, $46 \%$ of patients described paravertebral, nonradiating discomfort, unaccompanied by frequency-following muscle contractions. This was observed most commonly at the T-8 and T-9 levels. The effect was ameliorated in some cases by using multiple cathodes in parallel, which reduced the current density at individual contacts.

In 10 of these patients, this side effect interfered with an otherwise successful trial to such a degree that an insulated plate electrode was selected for permanent implantation at the same level as the percutaneous electrode. This eliminated the uncomfortable side effect completely in all patients. Intraoperative testing with the insulated plate electrode inverted (that is, contacts dorsal) reproduced the side effect in the majority of patients, indicating that structures dorsal to the electrode, such as fibers in ligamentum flavum, mediate the effect. Postoperative testing by computerized techniques demonstrated improved coverage of pain by stimulation paresthesias, with lower stimulation power requirements, using the insulated plate electrode.

This technical limitation of percutaneous spinal cord stimulation electrodes, which may confound treatment if unrecognized, has been found to have a straightforward solution which indicates the underlying mechanism.

Key Words * chronic pain * electrical stimulation * spinal cord * low-back pain

The use of percutaneous electrodes for spinal cord stimulation (SCS) was introduced in the 1970s as a method of screening patients for implantation of permanent electrodes.[3] By comparison with plate 
electrodes requiring laminotomy or laminectomy (Fig. 1), percutaneously inserted electrodes are minimally invasive; they reduce procedure-related discomfort that may compromise a therapeutic trial and that may itself represent a significant problem in patients with preexisting chronic pain. Percutaneous electrodes allow longitudinal access to multiple levels via a single interlaminar needle puncture, facilitating mapping of multiple targets. The use of temporary electrodes for an SCS trial offers major advantages in screening candidates for implantation of permanent devices.

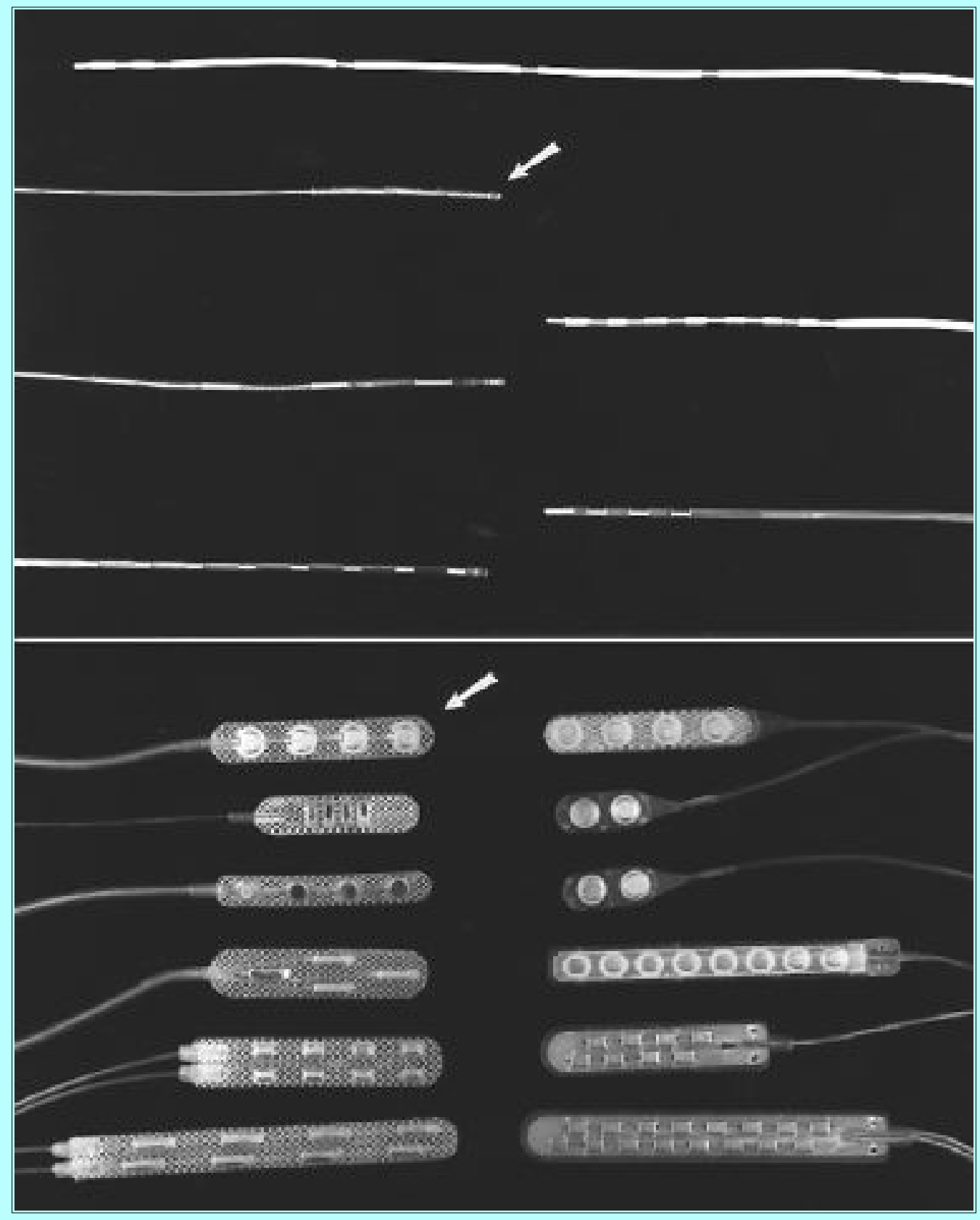

Fig. 1. Representative contemporary SCS designs from two manufacturers (Medtronic, Inc., Minneapolis, MN, left-hand columns of both figures; and Quest ANS [formerly Neuromed], Inc., Allen, TX, right-hand columns). Upper: Of the many available percutaneous designs, the model 3487A Pisces Quad used for this study (arrow) is prototypical. Lower: Of the many available plate designs, the model 3587A Resume used for this study (arrow) is prototypical.

Spinal cord stimulation is limited by the uncomfortable side effects experienced by patients as the amplitude of stimulation is increased. These side effects include pain referred to the dorsal spine, which 
may be attributable to dorsal root recruitment.[9] Alternatively, the pain may reflect recruitment of fibers in the ligamentum flavum or dura, which are closer to the electrodes, and therefore are exposed to higher current densities sufficient to recruit smaller fibers. Unlike plate electrodes with insulated backing, percutaneous electrode designs allow current to pass dorsally; they are radially symmetrical, with cylindrical spacers and contacts that are exposed circumferentially (Figs. 1 and 2). These design differences have been capitalized on in the present study to characterize this side effect and elucidate its mechanisms.

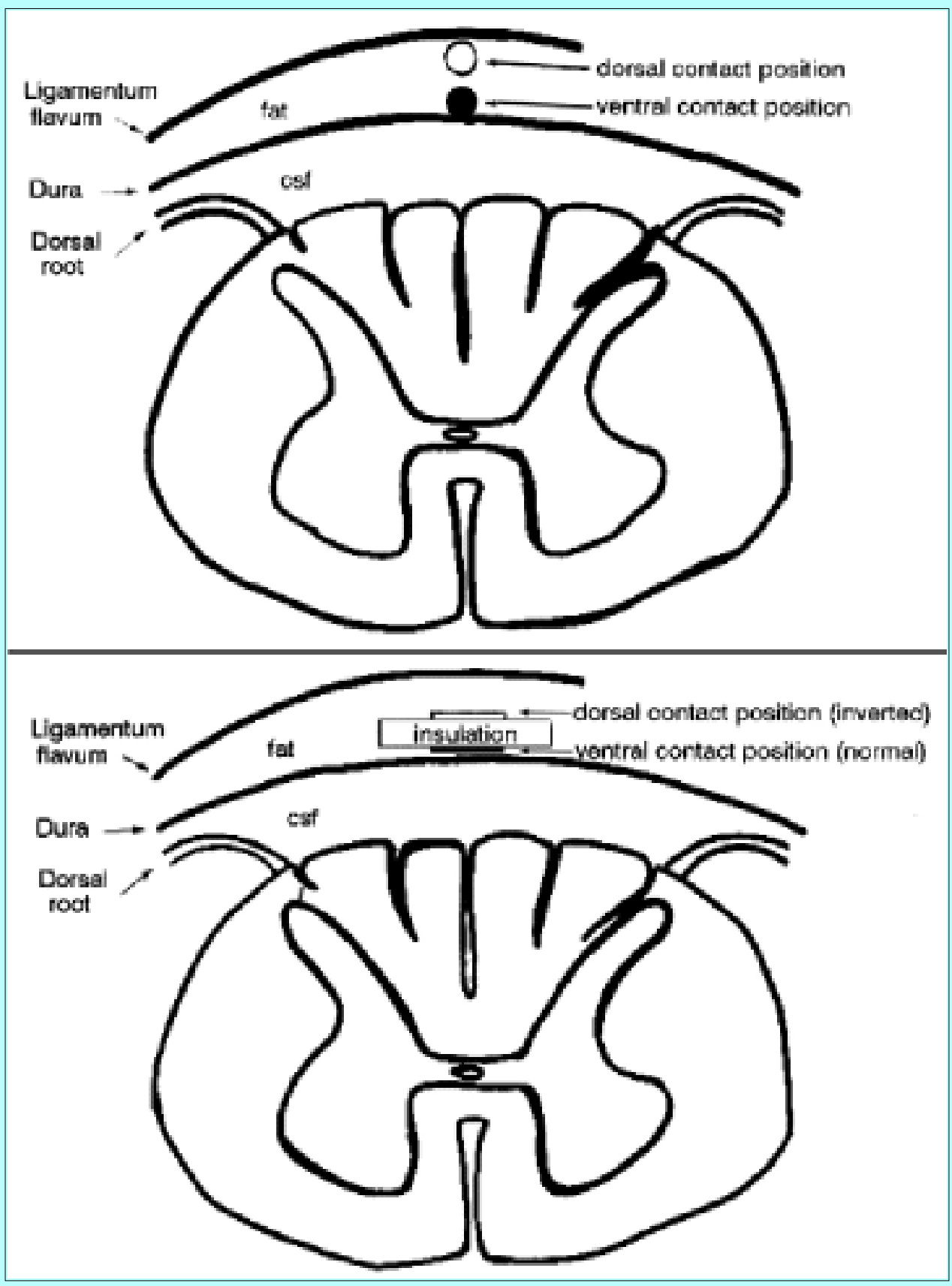

Fig. 2. Cross section of spinal canal and cord showing placement of stimulating electrode contacts and insulation. Upper: Percutaneously placed electrodes may pass ventrally (preferred) or dorsally within epidural fat adjacent to dura (preferred) or ligamentum flavum. Lower: Plate electrodes are implanted under direct vision adjacent to the dura with contacts ventral to insulation. For study purposes, they were tested briefly in an inverted position, with contacts dorsal to insulation. 


\section{CLINICAL MATERIALS AND METHODS}

A consecutive series of 79 candidates for SCS with postsurgical lumbar pain syndromes were screened using percutaneous four-contact electrodes (model 3487A; Medtronic, Inc., Minneapolis, MN) and tested at levels from T-8 through T-12. Electrodes were placed using fluoroscopic guidance; electrodes were steered along the radiographic midline in prone, awake patients after administration of local anesthesia. Only if bilateral paresthesias were achieved at or below $110 \%$ of the perceptual threshold was the electrode placement accepted as physiologically midline. The distributions of stimulation-evoked paresthesias as well as any stimulation-evoked discomfort were recorded systematically as a function of increasing amplitude. Both perceptual and bilateral thresholds as well as "usage" amplitude (producing broad but still comfortable stimulation paresthesias, overlapping the patient's usual distribution of pain) were tested.

Patients were blind to the position of the electrode (inverted vs. usual; longitudinal position) and the parameters of stimulation during all phases of testing in both temporary and permanent electrode placement. Stimulation pulse parameters were routinely set to 60 per second, with a $500-\mu$ sec pulse width. Routine contact assignments for intraoperative testing were bipolar with adjacent contacts on the electrode array active; cathode cephalad and anode caudad. When a patient reported stimulation-evoked discomfort, testing was repeated with multiple cathodes in parallel to reduce the current density at each cathode. The pulse repetition rate was then lowered to between 5 and 10 per second to allow observation of frequency-following muscle contractions.

Following temporary percutaneous electrode implantation, all patients underwent a clinical trial lasting at least 3 days. Those patients electing permanent electrode implantation were offered insulated plate electrodes (model 3587A; Medtronic Inc.) if stimulation-evoked discomfort had compromised percutaneous electrode placement at a level otherwise optimal for achieving the desired overlap of pain by stimulation paresthesias. The remaining patients undergoing permanent implantation received percutaneous electrodes (model 3487A; Medtronic, Inc.). Figure $1 \mathrm{a}$ and $\mathrm{b}$ shows the electrodes used for this study, and Table 1 presents their specifications.

\begin{tabular}{|ccc|}
\hline \multicolumn{4}{c|}{ TABLE 1 } \\
PERCUTANEOUS AND PLATE ELECTRODE SPECF KATIONS \\
\hline \hline & Percutane ous Model & Plate Model \\
Specification & $3487 \mathrm{~A}$ & $3587 \mathrm{~A}$ \\
\hline contact spacing & Pisces Quad & Resume \\
contact length & $9 \mathrm{~mm}$ & $10 \mathrm{~mm}$ \\
contact width & $3 \mathrm{~mm}$ & $4 \mathrm{~mm}$ \\
contact area & $1.2 \mathrm{~mm}$ & $4 \mathrm{~m} \mathrm{~m}$ \\
insulated backing & $11.3 \mathrm{~mm}$ & $12.5 \mathrm{~mm}$ \\
\hline & no & yes \\
\hline
\end{tabular}

Intraoperative test stimulation was conducted using the same methods as were used for temporary electrode placement. During implantation of the plate electrode via laminotomy, test stimulation was delivered with the electrode temporarily inverted (contacts dorsal) as well as in the usual position (contacts ventral, insulation dorsal). Figure 2 shows these parameters schematically.

Following placement of each electrode, computerized testing methods were used to characterize all 50 contact combinations for the four-contact array: these were presented to the patient in random order and a 
double-blind fashion. The patient-interactive system used for this study has been described previously.[6,7] All tests were performed with the patient sitting upright or slightly recumbent. The combinations affording the best clinical performance (per patient rating of overlap at usage threshold) were tested in greater detail; the thresholds of first perception, bilateral perception, low-back coverage, and discomfort were all recorded, along with graphic data that was entered directly by each patient. Graphic representation of the location of stimulation paresthesias was analyzed quantitatively, calculating overlap with areas of pain, which were entered by the patients using a digitizing tablet.

\section{RESULTS}

Forty-six percent of the 79 patients described paravertebral, nonradiating discomfort, unaccompanied by frequency-following muscle contractions at one or more levels during percutaneous electrode placement. This was observed most commonly at the T-8 and T-9 levels. Figure 3 shows the distribution observed by spinal level. When stimulation-evoked discomfort was observed, it was ameliorated by using multiple cathodes in parallel to reduce current density at individual contacts.

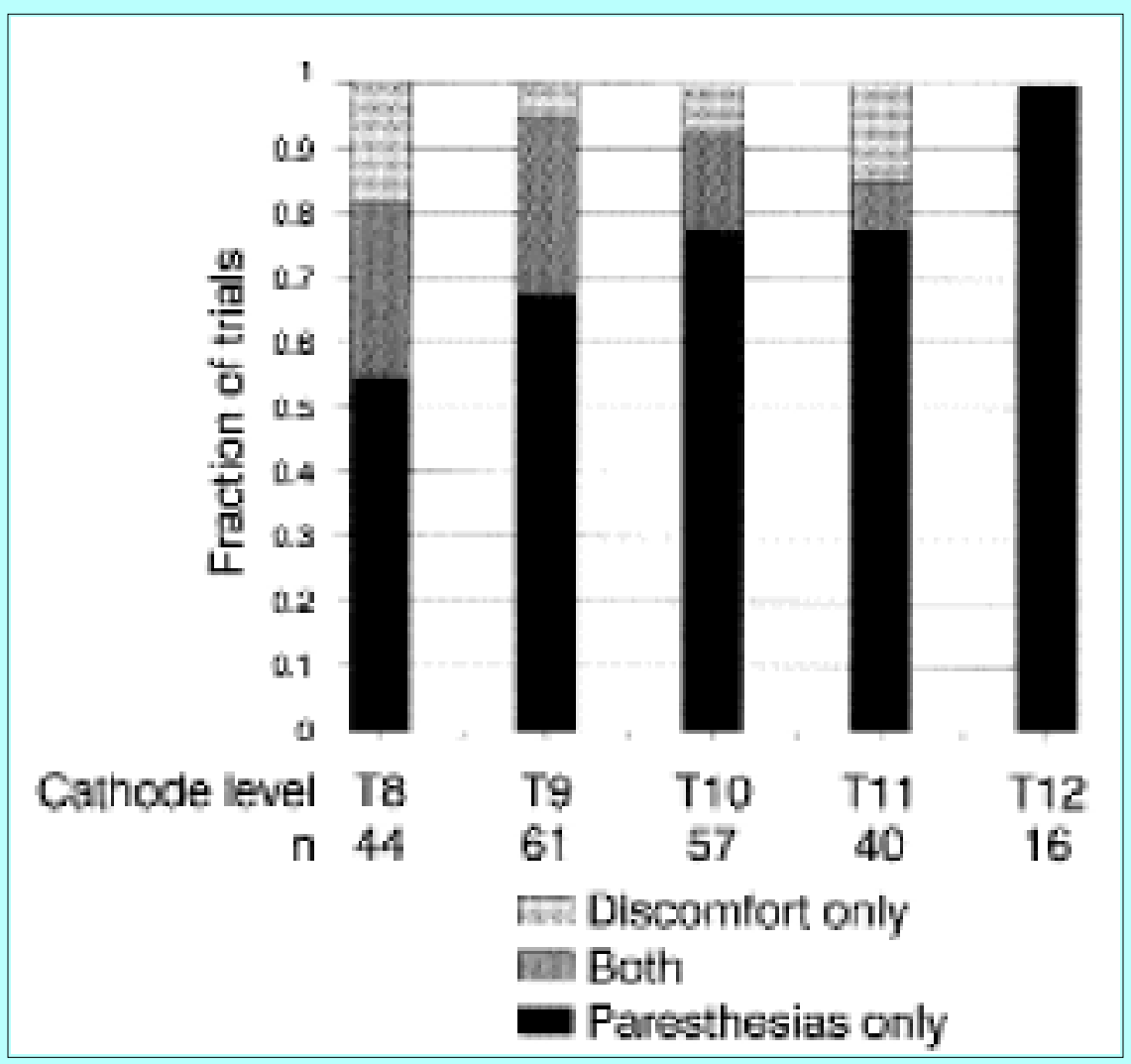

Fig. 3. Histogram showing the frequency of stimulation-evoked discomfort as a function of vertebral level. Forty-six percent of patients experienced this effect at some time, with the highest incidence at the T-8 and T-9 levels.

In 10 of the 79 patients, stimulation-evoked discomfort interfered to such a degree with an otherwise successful trial that an insulated plate electrode was selected for permanent implantation at the same level as the percutaneous electrode. Intraoperative testing with the insulated plate electrode temporarily inverted (that is, contacts dorsal) reproduced the side effect in seven patients at or below the amplitudes producing the side effect with the percutaneous electrode. Testing with the electrode in its normal position (contracts ventral) never did so. Postoperative testing with the permanently implanted electrode demonstrated no stimulation-evoked midback discomfort in any patient. 
Detailed quantitative measurements were made in nine patients with insulated plate electrodes placed at the same vertebral level as previous percutaneous electrodes (Table 2). The 10th patient received a "totally implanted" pulse generator (Itrel; Medtronic, Inc.), which is not supported by our computerized test equipment.

\begin{tabular}{|c|c|c|c|c|c|}
\hline AVERAGES & $\begin{array}{l}\text { FIVE } \mathrm{YA} \\
\text { PL }\end{array}$ & $\begin{array}{l}\text { TABLE } 2 \\
\text { ES, PERCL } \\
\text { E ELECTR }\end{array}$ & $\begin{array}{l}\text { TANEOUS YE } \\
D E^{\star}\end{array}$ & RSUS INS & ATED \\
\hline Plate Type & $\begin{array}{l}\text { Overlap } \\
\text { Rating }\end{array}$ & $\begin{array}{c}\text { Overlap } \\
\text { Area }\end{array}$ & $\begin{array}{c}\text { Extraneous } \\
\text { Area }\end{array}$ & $\begin{array}{l}\text { Symmetry } \\
\text { Sc ore }\end{array}$ & $\begin{array}{c}\text { Puse } \\
\text { Amplitude }\end{array}$ \\
\hline $\begin{array}{c}\text { peroutaneous } \\
(1 \times 4)\end{array}$ & $81 \pm 18$ & $45 \pm 31$ & $58 \pm 25$ & $82 \pm 24$ & $30 \pm 15$ \\
\hline $\begin{array}{l}\text { insulated } \\
(1 \times 4)\end{array}$ & $81 \pm 17$ & $58 \pm 32$ & $59 \pm 26$ & $85 \pm 21$ & $23 \pm 15$ \\
\hline $\begin{array}{l}{ }^{*} \text { Patients ente } \\
\text { and they entere } \\
\text { ous stimulation } \\
\text { paresthesias. T } \\
\text { of } 50-\% \text { of } \\
\text { nominal } 10-v \text { ot } \\
\text { de viation. }\end{array}$ & $\begin{array}{l}\text { gs of th } \\
\text { gs of bo } \\
\text { the topo } \\
\text { etrysool } \\
\text { either si } \\
\text { values }\end{array}$ & $\begin{array}{l}\text { aphy of } \\
\text { mas calc } \\
\text { of body } \\
\text { scaled } t\end{array}$ & $\begin{array}{l}\text { pain by st } \\
\text { r calculat } \\
\text { in, and s' } \\
\text { ted as } 10 \\
\text { he amplit } \\
\text { to } 100 \text {. }\end{array}$ & $\begin{array}{l}\text { mulation } \\
\text { n of o ierl } \\
\text { mmetry } \\
-2 \times \text { ab } \\
\text { de is a } p \\
\text { sitminus }\end{array}$ & $\begin{array}{l}\text { resthe sias } \\
\text { p, extrane- } \\
\text { stimulation } \\
\text { olute value } \\
\text { centage of } \\
\text { e standard }\end{array}$ \\
\hline
\end{tabular}

We observed a $29 \%$ improvement in calculated overlap of pain by stimulation paresthesias, with a $23 \%$ reduction in voltage requirement for the insulated plate electrode. These differences were not tested statistically because of the nonrandomized design of the study and the small sample size (nine patients).

\section{DISCUSSION}

We have characterized a technical limitation of percutaneous SCS electrodes, which may confound treatment of chronic lumbosacral pain syndromes. At thoracolumbar levels, there are differences in clinical performance of percutaneous and plate electrodes. Stimulation-evoked discomfort occurs in $46 \%$ of patients in whom percutaneous electrodes have been implanted, some time during testing while they are in the prone position, at amplitudes below those producing frequency-following segmental muscle contractions. This does not occur, in our experience, in the SCS literature, or in this study, with insulated plate electrodes, unless they are inverted.[1]

The stimulation-evoked discomfort observed here is to be distinguished from effects of dorsal root recruitment, which is predicted by finite element models of SCS to occur at relatively low thresholds, near perceptual threshold, with thoracic epidural electrodes.[9] Such recruitment is clinically recognizable as a radicular sensation and/or frequency-following intercostal or segmental reflex muscle contractions, which are palpable or visible at low-pulse repetition rates. The side effect at issue here is described by patients as well localized and nonradiating; it is similar to the effects of test stimulation with the electrode outside the spinal canal in ligament or paravertebral muscle (except that the latter will produce frequency-following motor responses).

The dorsal cerebrospinal fluid (CSF) thickness is at its maximum between dura-arachnoid and pia-arachnoid when the patient is prone, and so the amplitude necessary to stimulate the spinal cord using dorsal epidural electrodes is maximal.[2] Accordingly, the incidence of amplitude-dependent side effects may be expected to be maximal during electrode placement when the patient is prone, as opposed to upright or supine, when the device will generally be used clinically. Indeed, while using temporary percutaneous electrodes for clinical trials, only $13 \%$ of our patients reported prominent side effects. 
The incidence of stimulation-evoked discomfort as a function of vertebral level parallels CSF thickness, which determines amplitude threshold.[2] With increasing CSF thickness, the potential for dorsal root recruitment increases disproportionately.[9] The potential for this side effect may exist at all levels, but we observed it primarily at thoracic levels, where higher amplitudes are required (or tolerated).

Dividing current among multiple contacts of a percutaneous electrode often overcomes this problem by reducing current density locally. This is additional evidence for recruitment of structures dorsal to the electrode, as opposed to dorsal roots, which are more likely to be recruited with longer dipoles.[9] This measure, however, sacrifices some of the technical advantages of a multicontact array. The number of useful contact combinations is reduced, and the power requirements are increased, compromising the longevity of an implanted battery.

In the subset of 10 patients most severely affected, we found insulated plate electrodes at the same anatomical level completely eliminated this side effect of percutaneous electrodes. In no case was this side effect observed with insulated plate electrodes in the usual configuration, with contacts ventral. Furthermore, overlap of chronically painful areas by stimulation paresthesias was improved.

Insulated plate electrode placement not only has provided a straightforward solution to the clinical problem, but also has allowed testing that has indicated the underlying mechanism. The evoked discomfort is consistent with recruitment of pain-signaling fibers in ligamentum flavum dorsal to the electrode, because it occurs in most patients when the plate electrode is tested in an inverted position.

The insulating substrate of the plate electrode not only isolates the contact from dorsal structures, but because its cross-sectional area exceeds that of the percutaneous electrode, it also moves the contact surface ventrally, closer to the spinal cord. This may be expected to ameliorate unwanted dorsal root stimulation effects.[2] Because it is placed under direct vision, it is in direct contact with the dura; percutaneous electrodes placed with the use of fluoroscopic guidance may ascend within or behind epidural fat.

The insulated plate electrode substantially improved calculated overlap of pain by stimulation paresthesias, while reducing voltage requirements. "Totally implanted" systems with internal batteries may be expected to have improved longevity with insulated plate electrodes.

An additional potential advantage of plate electrodes is their resistance to longitudinal migration; the size of the plate exceeds the size of the connecting lead or cable, precluding movement after the wound heals and the electrode is encapsulated. Because of their cylindrical shape, percutaneous electrodes have a long-term potential for migration, even if encapsulated in scar tissue. Independently inserted, single contacts were found to have a high rate of migration, individually or with respect to one another, which necessitated frequent surgical revision of early systems;[5] but recent multicontact versions and contemporary anchoring techniques have ameliorated these problems.[4,8]

We were unable to reproduce stimulation-evoked discomfort in three of 10 patients with inverted laminotomy electrodes. Several mechanisms are possible. 1) The offending percutaneous electrode may have been dorsal to epidural fat; more ventral placement of either a percutaneous or plate electrode might have been sufficient to address this. Epiduroscopy or biplane fluoroscopy may be helpful. 2) The small laminotomy and midline removal of ligamentum flavum necessary to introduce a plate electrode may have ablated or altered the neural substrate of the side effect. 3) Current density around the exposed contact may have been too low compared with the percutaneous electrode. 4) Dorsal root stimulation 
may have been the mechanism in some patients: the absence of overt radicular effects and frequency-following responses notwithstanding. Finite element modeling of the inverted plate electrode would be of interest.

\section{CONCLUSIONS}

Plate electrodes are technically superior to percutaneous electrodes in patients with stimulation-evoked discomfort attributable to recruitment of local structures dorsal to the electrode. It remains to be shown that this is of lasting clinical value; this awaits long-term follow-up evaluation of these patients, none of whom, to date, have reported recurrent stimulation-evoked discomfort. The improved coverage and system efficiency observed with plate electrodes in these patients may or may not be demonstrable in patients in general. A prospective, randomized comparison study of percutaneous and plate electrodes is underway.

\section{References}

1. Barolat G, Massaro F, He J, et al: Mapping of sensory responses to epidural stimulation of the intraspinal neural structures in man. J Neurosurg 78:233-239, 1993

2. Holsheimer J, den Boer JA, Struijk JJ, et al: MR assessment of the normal position of the spinal cord in the spinal canal. AJNR 15:951-959, 1994

3. Hoppenstein, R: Percutaneous implantation of chronic spinal cord electrodes for control of intractable pain: preliminary report. Surg Neurol 4:195-198, 1975

4. North RB, Ewend MG, Lawton MT, et al: Spinal cord stimulation for chronic, intractable pain: superiority of "multi-channel" devices. Pain 44:119-130, 1991

5. North RB, Fischell TA, Long DM: Chronic stimulation via percutaneously inserted epidural electrodes. Neurosurgery 1:215-218, 1977

6. North RB, Fowler K, Nigrin DJ, et al: Automated 'pain drawing' analysis by computer-controlled, patient-interactive neurological stimulation system. Pain 50:51-58, 1992

7. North RB, Fowler K, Nigrin DJ, et al: Patient-interactive, computer-controlled neurological stimulation system: clinical efficacy in spinal cord stimulator adjustment. J Neurosurg 76:976-972, 1992

8. North RB, Kidd DH, Zahurak M, et al: Spinal cord stimulation for chronic, intractable pain: experience over two decades. Neurosurgery 32:384-395, 1993

9. Struijk JJ, Holsheimer J, Boom HB: Excitation of dorsal root fibers in spinal cord stimulation: a theoretical study. IEEE Trans Biomed Eng 40:632-639, 1993

Manuscript received November 21, 1996.

Accepted in final form December 18, 1996.

Address reprint requests to:Richard B. North, M. D., Department of Neurosurgery, Johns Hopkins University School of Medicine, 600 North Wolfe Street, Meyer 7-113, Baltimore, Maryland 21287-7713. 